\title{
RESEARCH
}

Open Access

\section{Probiotic Bifidobacterium strains and galactooligosaccharides improve intestinal barrier function in obese adults but show no synergism when used together as synbiotics}

Janina A. Krumbeck ${ }^{1}$, Heather E. Rasmussen², Robert W. Hutkins ${ }^{1 *}$, Jennifer Clarke ${ }^{1}$, Krista Shawron ${ }^{3}$, Ali Keshavarzian ${ }^{3 *}$ and Jens Walter ${ }^{1,4,5,6^{*}}$ (D)

\begin{abstract}
Background: One way to improve both the ecological performance and functionality of probiotic bacteria is by combining them with a prebiotic in the form of a synbiotic. However, the degree to which such synbiotic formulations improve probiotic strain functionality in humans has not been tested systematically. Our goal was to use a randomized, double-blind, placebo-controlled, parallel-arm clinical trial in obese humans to compare the ecological and physiological impact of the prebiotic galactooligosaccharides (GOS) and the probiotic strains Bifidobacterium adolescentis IVS-1 (autochthonous and selected via in vivo selection) and Bifidobacterium lactis BB-12 (commercial probiotic allochthonous to the human gut) when used on their own or as synbiotic combinations. After 3 weeks of consumption, strain-specific quantitative real-time PCR and $16 \mathrm{~S}$ rRNA gene sequencing were performed on fecal samples to assess changes in the microbiota. Intestinal permeability was determined by measuring sugar recovery in urine by GC after consumption of a sugar mixture. Serum-based endotoxin exposure was also assessed.

Results: IVS-1 reached significantly higher cell numbers in fecal samples than BB-12 $(P<0.01)$ and, remarkably, its administration induced an increase in total bifidobacteria that was comparable to that of GOS. Although GOS showed a clear bifidogenic effect on the resident gut microbiota, both probiotic strains showed only a non-significant trend of higher fecal cell numbers when administered with GOS. Post-aspirin sucralose:lactulose ratios were reduced in groups IVS-1 $(P=0.050)$, IVS-1 + GOS $(P=0.022)$, and GOS $(P=0.010)$, while sucralose excretion was reduced with BB-12 $(P=0.002)$ and GOS $(P=0.020)$, indicating improvements in colonic permeability but no synergistic effects. No changes in markers of endotoxemia were observed.
\end{abstract}

\footnotetext{
*Correspondence: rhutkins1@unl.edu; ali_keshavarzian@rush.edu; jwalter1@ualberta.ca

'Department of Food Science and Technology, 258 Food Innovation Center Lincoln, University of Nebraska-Lincoln, Lincoln, NE 68588, USA

${ }^{3}$ Division of Digestive Diseases and Nutrition, Department of Internal

Medicine, Rush University Medical Center, Chicago, IL, USA

Full list of author information is available at the end of the article
}

(c) The Author(s). 2018 Open Access This article is distributed under the terms of the Creative Commons Attribution 4.0 International License (http://creativecommons.org/licenses/by/4.0/), which permits unrestricted use, distribution, and reproduction in any medium, provided you give appropriate credit to the original author(s) and the source, provide a link to the Creative Commons license, and indicate if changes were made. The Creative Commons Public Domain Dedication waiver (http://creativecommons.org/publicdomain/zero/1.0/) applies to the data made available in this article, unless otherwise stated. 
(Continued from previous page)

Conclusion: This study demonstrated that "autochthony" of the probiotic strain has a larger effect on ecological performance than the provision of a prebiotic substrate, likely due to competitive interactions with members of the resident microbiota. Although the synbiotic combinations tested in this study did not demonstrate functional synergism, our findings clearly showed that the pro- and prebiotic components by themselves improved markers of colonic permeability, providing a rational for their use in pathologies with an underlying leakiness of the gut.

Keywords: Synbiotic, Probiotic, Prebiotic, Obesity, Gut barrier function, Autochthonous, Allochthonous, Galactooligosaccharide, Bifidobacteria, Bifidobacterium

\section{Background}

It is now well established that the gastrointestinal (GI) microbiota has a fundamental impact on metabolic, immunological, and endocrine functions of the host [1-4]. The host-microbe interrelationship is viewed as mutualistic, contributing to overall host health [5]. However, aberrations in both microbiota composition and function can result in the development of several chronic disease states [6-8]. Obesity, in particular, is associated with a dysbiosis characterized by low diversity and specific shifts in bacterial taxa that correlate with metabolic and inflammatory markers [9]. Although causation has not yet been established in humans, these associations suggest that gut microbes contribute to the subclinical systemic inflammation that ultimately lead to the development of pathologies such as insulin resistance, type- 2 diabetes, and cardiovascular disease $[10,11]$. One mechanism by which the gut microbiota contributes to systemic inflammation is through the translocation of pro-inflammatory and immunogenic bacterial compounds, including lipopolysaccharide (LPS) and peptidoglycan, that can drive metabolic endotoxemia [12, 13]. In fact, intestinal permeability has been shown to be elevated in at least a subset of obese subjects and is increased by high-fat diets, potentially constituting a mechanism by which the microbiota contribute to obesity [14]. The microbiome's influence on both the development of endotoxemia as well as intestinal barrier integrity makes it a rational target for dietary strategies aimed at reducing intestinal epithelial permeability and obesity-associated pathologies.

Data from animal and in vitro studies suggest that both specific bacterial taxa and their metabolic products influence intestinal barrier function. For example, short-chain fatty acids produced from the fermentation of non-digestible carbohydrates (including commonly used prebiotics) have been reported to improve expression of tight junction proteins, such as zona occludens proteins (ZO-1-2), occluding, and claudin 1-4 (CLDN1-4) [15-19]. In addition, several studies suggest that probiotics, including Bifidobacterium spp., Lactobacillus spp., and other lactic acid bacteria, were associated with barrier function integrity in vitro and in vivo [20-25]. In addition to strong associations between Bifidobacterium numbers and improvements in intestinal epithelial cell barrier function and intestinal permeability [12, 26, 27], functional studies have begun to establish a causative role. For example, a strain of Bifidobacterium longum subsp. infantis (recently reclassified as Bifidobacterium animalis subsp. lactis [28]) increased trans-epithelial resistance and expression of tight junction proteins in IL-10-deficient mice [29] and decreased intestinal permeability in mice suffering from necrotizing enterocolitis [30]. Furthermore, treatment with B. infantis and Bifidobacterium bifidum decreased the gut endotoxin concentration in mice [31], and Bifidobacterium adolescentis administration to rats significantly lowered rates of bacterial translocation [32]. Bifidobacteria have also been associated with metabolic improvements considered to be associated with inflammation, including insulin sensitivity, white fat accumulation, liver weight [33], reactive oxygen species, nuclear factor $\mathrm{\kappa B}$ activation, and reduced markers of inflammation [34], and high-density lipoprotein (HDL) plasma cholesterol levels [35].

These findings provide a rational basis for the development of strategies intended to enrich for Bifidobacterium populations in the human gut. This can be achieved through dietary consumption of probiotics and prebiotics. The consumption of prebiotic carbohydrates, such as galactooligosaccharide (GOS), resistant starch, fructooligosaccharides (FOS), and inulin, have been shown to increase autochthonous bifidobacteria in infants [36-38] and adults [39-44]. However, the relative abundance of resident Bifidobacterium levels in adults is highly variable, ranging from 0 to $3 \%$ [45-48], and not all subjects respond to prebiotic intervention, even at high doses [45, $46,49,50]$. Therefore, one approach to enrich for bifidobacteria, increase the number of responders, and enhance their metabolic activity would be to administer a prebiotic together with a select probiotic Bifidobacterium strain or strains that use the prebiotic as a growth substrate in vivo. Such pairings are referred to as synergistic synbiotics [51]. According to ecological theory, the provision of resources in a microbial community leads to a relaxation of competition [52, 53] and therefore could enhance colonization success of probiotic strains $[52,53]$.

Several recent studies have reported improvements in specific health biomarkers or outcomes after consumption 
of synbiotics [54-56]. Few studies, however, have systematically determined if synbiotics improve the ecological attributes (such as establishment) and/or enhance the health benefits of specific probiotics strains compared to the probiotic alone [57-62]. Moreover, it is currently unknown if it is possible for a probiotic strain to benefit from the presence of a prebiotic substrate in the competitive environment of the human gut. Stable engraftment of an autochthonous $B$. longum strain in the human gut was detected in subjects with an apparent open niche based on resources [63], but it is unclear if such substrates can be administered by the diet. It is also possible for prebiotics to exert microbiota-independent effects [64-66].

We have recently developed a method for the selection of autochthonous bacterial strains that are able to benefit from prebiotic substrates in the competitive environment of the gastrointestinal tract [61]. This approach, in vivo selection (IVS), is based on the identification of bacterial strains that became enriched in human fecal samples through the administration of a prebiotic compound, providing a high likelihood that the strain can preferentially utilize the substrate under the exact ecological condition that prevail in the human gut. One such strain, $B$. adolescentis IVS-1, was isolated from a human subject, was unique to that subject, and grew well on GOS $[45,61]$. Remarkably, when B. adolescentis IVS-1 was fed to rats, its relative abundance increased from $3 \%$ in the absence of GOS to $37 \%$ when fed as a synbiotic, i.e., in the presence of GOS [61]. The ability of IVS-1 to expand more than tenfold, even in a different host animal, suggested that this strain could be similarly enriched in human subjects.

The primary goal of this study, therefore, was to systematically compare the ecological and functional properties of a rationally selected synergistic synbiotic in a parallel-arm, placebo-controlled human trial. Treatments included the human autochthonous strain, $B$. adolescentis IVS-1, paired with its cognate prebiotic (GOS), as well as a GOS synbiotic containing an allochthonous commercial strain, $B$. animalis subsp. lactis BB-12 [67]. The latter is known to utilize GOS in vitro and indeed has a higher growth rate on GOS than on glucose $[68,69]$. This strain has also been combined with GOS and used previously as a synbiotic in human trials [70-72]. We also included treatments containing only the probiotic strain or the prebiotic. This study design allowed us to assess the ability of the probiotic strains to establish in the gastrointestinal tract, in the presence and absence of GOS, and to identify their effects on microbiota composition. Clinical outcomes were also assessed, with gut permeability as the primary endpoint in a target group susceptible to a leaky gut (obese individuals).

\section{Methods \\ Subjects}

This study was a randomized, double-blinded, placebocontrolled, parallel-arm clinical trial conducted at Rush University Medical Center (RUMC) in Chicago, USA. Women and men between 18 and 65 years with a BMI of $30.0-40.0 \mathrm{~kg} / \mathrm{m}^{2}$ were recruited. Exclusion criteria included the following: (1) prior intestinal resection; (2) patient history of GI diseases except for hiatal hernia, gastroesophageal reflux disease (GERD), and hemorrhoids; (3) severe renal disease defined by creatinine more than twice normal; (4) markedly abnormal liver function defined by ALT/AST over four times normal levels or elevated bilirubin; (5) antibiotic use within the last 12 weeks prior to enrollment; (6) lean or overweight $\left(\mathrm{BMI}<30.0 \mathrm{~kg} / \mathrm{m}^{2}\right)$, (7) intolerant to aspirin; (8) regular use of aspirin; (9) excessive alcohol intake (more than two drinks for men and one drink for women daily); (10) presence of chronic metabolic disease such as symptomatic cardiovascular disease, insulin-requiring or uncontrolled diabetes, current active treatment of cancer; (11) a plan to have a major change in dietary habit during the following 6 months; (12) consumption of probiotics, prebiotics, or synbiotics without an appropriate 2-week washout period; (13) self-reported lactose intolerance; (14) subjects younger than 18 or older than 65; and (15) unwillingness to consent to the study.

\section{Study design}

Four visits were required for each subject (Fig. 1a). At visit 1 , potential subjects were screened for eligibility and provided written informed consent. Vitals and anthropometrics were completed, and blood was obtained for endotoxin and metabolic markers. Subjects were instructed to collect stool in anaerobic bags before visit 2 and deliver samples within $24 \mathrm{~h}$ if stored at $-20{ }^{\circ} \mathrm{C}$ or within $5 \mathrm{~h}$ if stored at room temperature. At visit 2, study subjects completed a 3-day food record and a standardized 34-item GI symptom questionnaire (GSSC; Gastrointestinal Symptom and Severity Checklist) to identify potential impacts of treatment on GI symptoms, including stool consistency, discomfort, flatulence, abdominal pain, and bloating, on a scale from 0 (best) to 10 (worst). Within 1 week, subjects returned for visit 3 to provide urine for baseline intestinal permeability measurement, completed a blood draw, and were randomized to one of the six treatments (see below). At the end of a 3-week treatment period, subjects returned to the clinic to provide stool and urine samples and to complete a 3-day food record to ensure consistency of dietary intake throughout the study. A blood draw, anthropometrics, and identical follow-up questionnaires were completed. This study was approved by the Institutional Review Board at RUMC in February of 2012, and all procedures 


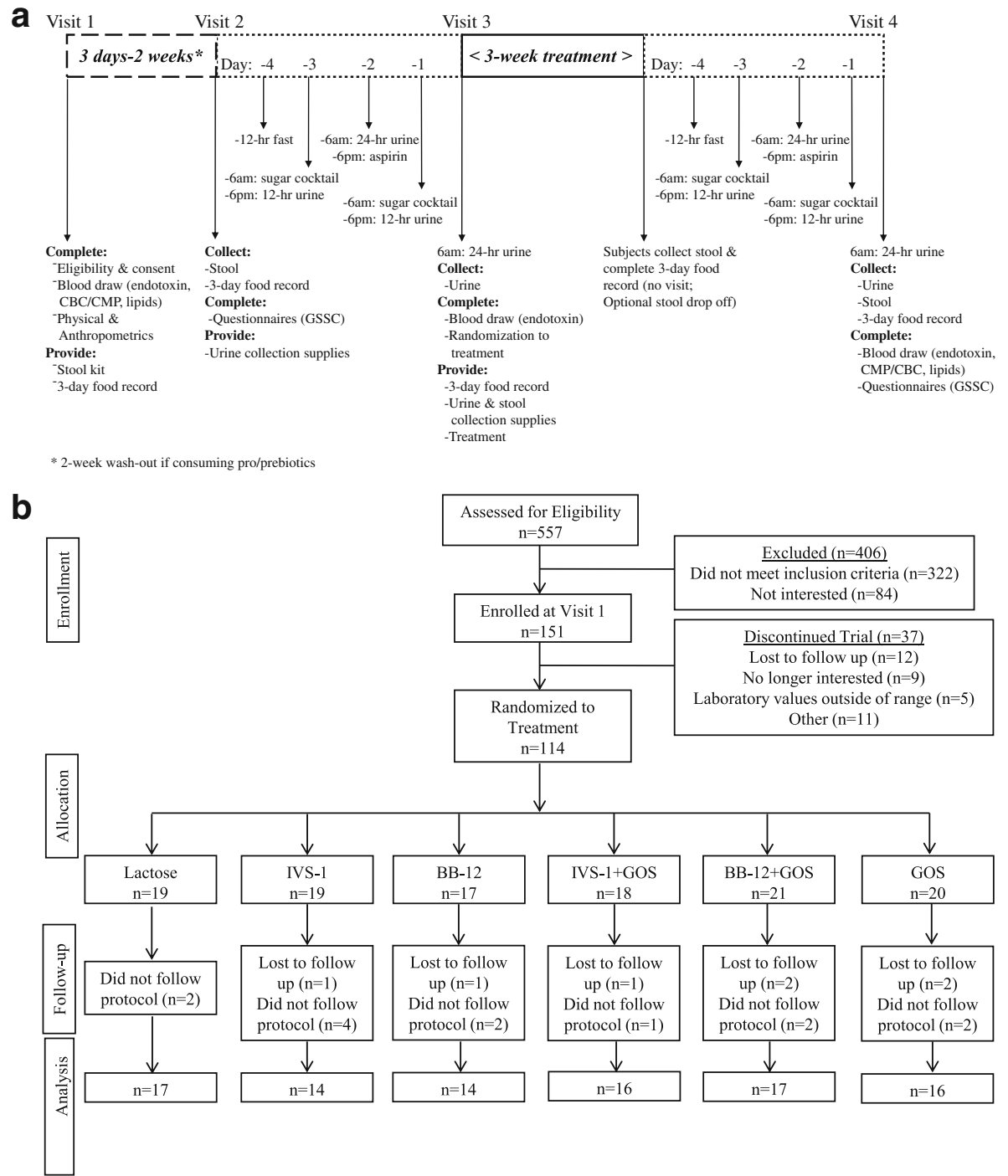

Fig. 1 Experimental design and participant flow diagram. a Time line for the human trial. Four visits were required from each subject. At visit 1 , potential subjects were screened for eligibility and provided with a 3-day food record, all supplies for stool and urine collection (stool kit, urine collection containers, sugar cocktail, and aspirin), and instructions for specimen handling and for completing these tasks before the next visit. Each subject collected stool before taking the sugar cocktail to avoid potential effects of sugar cocktail on microbiota composition. Details of the stool and urine collection are shown. GSSC, Gastrointestinal Symptom and Severity Checklist. b Participant flow diagram showing the progress through the phases of the randomized controlled trial (enrollment, intervention allocation, follow-up, and data analysis)

were conducted according to the principles expressed in the Declaration of Helsinki. This trial was registered at clinicaltrials.gov, identifier NCT02355210.

\section{Treatments}

The prebiotic, GOS, was obtained from FrieslandCampina, Amersfoort, the Netherlands (sold under the trade name, Vivinal GOS powder) and contained $72.5 \%$ GOS, $22.8 \%$ lactose, and $4.7 \%$ monosaccharides (galactose and glucose). It was previously established that a GOS dose of $5 \mathrm{~g}$ per day was sufficient to induce a bifidogenic response [39]. Therefore, the total amount of GOS powder was increased to $6.9 \mathrm{~g}$ to achieve a dose of $5 \mathrm{~g}$ GOS. An additional $0.1 \mathrm{~g}$ of lactose was added to achieve the same weight $(7.0 \mathrm{~g})$ as the other preparations.

The two probiotic strains used were $B$. adolescentis IVS-1 [61] and B. animalis subsp. lactis BB-12. Strain IVS-1 was produced from a contract manufacturer (Danwell Technology, Garden Grove, CA) and provided as a freeze-dried powder. Strain BB-12 was provided by Chr. Hansen (Hørsholm, Denmark), also as a high cell density freeze-dried powder. Its reported ability to ferment GOS was confirmed in preliminary experiments (data not shown). The probiotic powders were stored for up to 6 months at $-18{ }^{\circ} \mathrm{C}$, and viable cell numbers, as determined by cultural enumeration, were shown to 
be stable throughout the entire study period (data not shown). Probiotic treatments were portioned into sachets, each containing $0.1 \mathrm{~g}$ of probiotic powder $\left(10^{10} \mathrm{CFU} / \mathrm{g}\right)$, resulting in a daily dose of $1 \times 10^{9} \mathrm{CFU}$ and $6.9 \mathrm{~g}$ of lactose as a carrier/control. Synbiotics contained $6.9 \mathrm{~g}$ of Vivinal and $0.1 \mathrm{~g}$ of probiotic (either $B$. adolescentis IVS-1 or B. animalis BB-12), for a total dose of $7.0 \mathrm{~g}$. Placebo samples contained $7.0 \mathrm{~g}$ of lactose.

The material was packaged in sachets in the Food Processing Product Development Lab at the University of Nebraska. The sachet material was impermeable to oxygen and moisture. Subjects were provided with enough sachets for the entire length of the 3-week treatment period and were instructed to consume one sachet daily, with $2 \mathrm{~h}$ between treatment and food consumption in order to facilitate delivery of the probiotic/prebiotic compounds into the cecum/large intestine by rapid emptying of the compounds from the stomach and through small bowel. Subjects were instructed to mix the treatment with the provided bottled water only, as previous testing indicated reduction in bacterial count of IVS-1 with tap water (data not shown). All subjects were instructed to store treatments in their freezer $\left(-18{ }^{\circ} \mathrm{C}\right)$.

\section{Analysis of fecal microbiota}

Fecal samples were stored in aliquots at $-80{ }^{\circ} \mathrm{C}$ until further analysis. The DNA was extracted as previously described [35]. Amplicon sequencing (Illumina MiSeq platform v3 kit producing 300-bp paired-end sequences) was performed at the University of Minnesota Genomics Center, with all samples being included in a single run. The V5-V6 region of the $16 \mathrm{~S}$ rRNA gene was amplified using primer pair 784F (5'-RGGATTAGAT ACCC-3') and 1064R (5'-CGACRRCCATGCANCACC T-3'). These primers were chosen based on our previous findings that the V5-V6 region provided the best resolution among Bifidobacterium species [61, 63], which we considered the most important criteria for the aims of our study. The 25- $\mu \mathrm{l}$ PCR mixtures contained $5 \mu \mathrm{l}$ of template DNA, $5 \mu \mathrm{l}$ of $2 \times$ HotStarTaq PCR master mix, a final

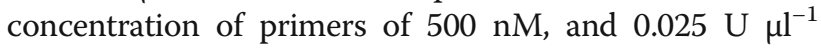
HotStarTaq polymerase (Qiagen Inc.), as previously described [61]. The generated sequences were quality filtered with Illumina software at the University of Minnesota Genomics Center, resulting in more than $96 \%$ of the samples meeting all quality control criteria. Sequences that did not meet quality filtering criteria were removed from the analysis. All reads were trimmed to 240 base pairs using the FASTX-Toolkit. The reads were merged and analyzed for their sequencing depth. If a sample exceeded 37,000 reads, it was subsampled using Mothur v.1.31.162, while samples that had less than 37,000 sequences were left untreated. This normalization step was done as suggested by Weiss et al. to account for differences in the library size and minimize potential biases due to sequencing depth across samples [73]. Subsequently, reads were filtered by length with a minimum of 240 base pairs and a maximum of 260 base pairs, dereplicated, OTU clustered, chimeras removed, and taxonomically assigned as previously described [61]. After processing and quality control, samples contained an average of $22,487.59 \pm 6683$ sequences.

\section{Quantitative real-time PCR (qPCR)}

qPCR was performed by using a Mastercycler Realplex2 instrument (Eppendorf AG, Hamburg, Germany). Each PCR was performed with 25 - $\mu$ l volumes using real-time master mix containing SYBR (5 Prime Inc., Gaithersburg, MD) and either genus-specific primers for Bifidobacterium [39], or the strain-specific primers for B. adolescentis IVS-1, as described previously [61]. Strain-specific PCR for IVS-1 did not reveal products in baseline samples in subjects of groups IVS-1 or IVS-1 + GOS or in the baseline of another additional 20 randomly selected subjects (data not shown).

For strain-specific detection of $B$. animalis subsp. lactis $\mathrm{BB}-12$, the PCR mixture contained $25 \mu \mathrm{l}$ of PCR reaction mix (QuantiFast ${ }^{\oplus}$ Probe PCR Kit, QIAGEN, Hilden, Germany), $0.3 \mu \mathrm{mol}$ of each primer (BAL-23S-F 5'-CA GGTGGTCTGGTAGAGTATACCG-3' and BAL-23S-R 5'-ACGGCGACTTGCGTCTTG-3'), $0.25 \mu \mathrm{mol}$ of probe (BAL-23S-P 5'-FAM-CGCCCACGACCCGCAAG-TAMR A-3'), and $5 \mu \mathrm{l}$ DNA as previously described [74]. The target of these primers and probe is the elongation factor $\mathrm{Tu}$ (tuf) gene of BB-12. The specificity of the primers and probe for BB-12 was validated experimentally by qPCR using DNA from 11 different Bifidobacterium strains using the same approach as described previously for the IVS-1 strain-specific qPCR [61]. These strains included B. adolescentis IVS-1, B. adolescentis ATCC 15703, B. adolescentis L2-32, B. longum subsp. longum ATCC 15707, B. longum DJO10A, B. longum ATCC 15697, B. longum subsp. longum F8, B. longum subsp. longum JDM301, Bifidobacterium sp. strain 113, Bifidobacterium sp. strain 12_1_47BFAA, and Bifidobacterium sp. strain HMLN14. Furthermore, primers were tested against fecal DNA obtained from baseline fecal samples from subjects in BB-12 and BB-12 + GOS groups, and additional randomly selected samples from other subjects. Strain BB-12 was detected in three out of 51 tested subjects before the treatment was started, which may be due to accidental consumption of food products containing this strain.

Overall, we concluded that both qPCR assays were sufficiently specific and that the test strains were either absent or rare in the pre-treatment microbiota of the subjects. Absolute quantification of both strains was achieved through standard curves prepared by tenfold dilutions of 
DNA isolated from overnight cultures $(14 \mathrm{~h})$ for which cell numbers were determined by quantitative culture.

\section{Intestinal permeability}

Subjects ingested a sugar mixture containing $2 \mathrm{~g}$ mannitol, $7.5 \mathrm{~g}$ lactulose, $40 \mathrm{mg}$ sucrose, and $2 \mathrm{~g}$ sucralose after a 12-h overnight fast (Fig. 1a). Subjects collected urine into three separate containers for 5,7 , and $12 \mathrm{~h}$, for a total collection time of $24 \mathrm{~h}$, in order to estimate gastroduodenal permeability ( $5 \mathrm{~h}$ urinary sucrose), proximal and distal small bowel permeability $(5 \mathrm{~h}$ and first $12 \mathrm{~h}$ urinary mannitol and lactulose and lactulose:mannitol ratio), and total and primarily colonic permeability (24 h urinary sucralose level and sucralose/ lactulose ratio) as we previously described [75]. Compared to normal-weight individuals, obese individuals are more likely to have a hyper-permeable intestine [76]. However, values vary greatly among individuals, and a treatment with aspirin has been shown to improve consistency in intestinal permeability measurements [76, 77]. Therefore, the subjects participated in an aspirin challenge consuming four $325-\mathrm{mg}$ aspirin tablets both $12 \mathrm{~h}$ before and immediately before ingestion of the sugar mixture. Thus, urine was collected for two separate 24-h periods with and without aspirin treatment, and both before and after treatment. Urine was analyzed for concentrations of mannitol, lactulose, and sucralose using gas chromatography (GC). Intestinal permeability was expressed as a percent of the oral dose excreted in the urine.

\section{Plasma and serum measures of endotoxin exposure}

Serum endotoxin (LPS) and LPS-binding protein (LBP) were measured after the aspirin challenge both before and after treatment. Endotoxin was measured in serum by Limulus Amebocyte Lysate QCL-1000 (Lonza \# 50-647U). Serum samples were diluted at a 1:5 ratio with LAL reagent water. LBP was measured in plasma using an ELISA kit from Cell Sciences Inc. (\# HK315).

\section{Serum metabolic markers}

A complete metabolic panel and complete blood count were performed to allow an assessment of treatment safety. To assess impact of treatments on metabolic markers, a lipid panel was completed by Quest Diagnostics and included the following: total cholesterol (TC), low-density lipoprotein cholesterol (LDL-C), high-density lipoprotein cholesterol (HDL-C), and triglycerides. Non-HDL was calculated by subtracting HDL-C from TC.

\section{Vitals and anthropometrics}

Blood pressure was measured using an automated cuff with the average of three assessments used for statistical comparisons. Body weight and waist circumference was assessed and BMI calculated before and after treatment.

\section{Statistical analysis}

All data presented was analyzed based on a per protocol analysis. Subjects were excluded from the analysis if the study protocol was not followed, including use of antibiotics during the treatment period, storage of the treatments at room temperature, and use of tap water to consume treatments. Data is presented as mean \pm SEM for variables that were normally distributed, or median (IQR) for variables not normally distributed. Group means were compared by ANOVA and post hoc tests except when data were not normally distributed, in which case a nonparametric analysis of medians was performed using the Kruskal-Wallis test. Chi-square tests or Fisher's exact tests were used for incidence data. If only two groups were compared, Student's $t$ tests were performed.

For the analysis of the gut microbiota, Mann-WhitneyWilcoxon matched pair tests were used for pairwise comparisons between time points for the 16S rRNA gene sequencing and qPCR data, as data was normally distributed. Unpaired Mann-Whitney-Wilcoxon tests were used for pairwise comparisons between treatment groups. When more than two samples were compared, Kruskal-Wallis test was applied. $P$ values were corrected for the total number of comparisons with the use of a false-discovery rate (FDR) method in R, whereby values were considered significant for FDR-adjusted $P$ values (reported as $Q$ values) of $<0.1$.

\section{Results}

\section{Subject enrollment}

A total of 151 volunteers were enrolled in the study (Fig. 1b). Of these, 114 subjects were randomly assigned to the six treatments, and 94 were used in the analyses after accounting for attrition and excluding for protocol deviation.

\section{Baseline demographic and clinical characteristics}

The majority of the subject cohort was female (71\%), middle aged $(44.3 \pm 11.2$ [mean $\pm \mathrm{SD}])$, non-Hispanic or Latino (90.4\%), and of African American ethnicity (61.7\%) (Additional file 1: Table S1). All subjects were obese, with a median (IQR) BMI of $36.7(8.5) \mathrm{kg} / \mathrm{m}^{2}$ and waist circumference of $45.0 \pm 7.3 \mathrm{in}$. Other clinical metabolic markers were within the normal range (Additional file 1: Table S1). Randomization resulted in all demographic and clinical characteristics, including race, ethnicity, systolic/diastolic blood pressure, cholesterol (total, LDL, HDL, non-HDL), glucose, and triglyceride, to not differ significantly between groups with one exception: the IVS-1 + GOS group had a higher BMI $(P=0.049)$ when compared to the IVS-1 group (Additional file 1: Table S1). 


\section{Safety and tolerability of treatments and impact on dietary patterns}

No differences in complete metabolic panel and complete blood count values were observed with any treatment (data not shown). Mild gastrointestinal symptoms are common among heathy obese adults, and many study participants reported bloating (60.6\%), passing gas (85.1\%), hard stools (46.8\%), and watery stools $(43.6 \%)$ at baseline. No significant differences in median symptom score were detected between the six groups at baseline (Additional file 1: Table S2). Treatments were generally well tolerated with minimal reported side effects. The GOS group had significantly harder stools when compared to the BB-12+ GOS group $(P=0.024)$. Passing gas increased from a median of 2.5 to 5.0 with lactose supplementation, potentially because of undeclared lactose intolerance, but this was not significantly different between baseline and treatment end $(P=0.150)$. The "severity of passing gas" was significantly reduced from 4.0 to 1.0 in the BB-12 + GOS group (comparison of baseline to treatment; $P=0.040)$, and severity of hard stools increased from 1.0 to 3.5 (on a score from 1 to 10 ) in the GOS group $(P=0.030)$. No differences in macronutrient or micronutrient intake were detected before and after the treatment period, suggesting treatment tolerability was not influenced by dietary intake (data not shown).

\section{Cell numbers of strains IVS-1 or BB-12 in fecal samples}

Strain-specific qPCR revealed that both strains reached significantly higher cell numbers during the treatment period when compared to the baseline samples $(P<0.001$ in group IVS-1 + GOS; $P<0.0007$ in groups IVS-1, BB-12, and BB-12+GOS) (Fig. 2a). IVS-1 was detectable at an average of $6.99 \pm 1.2 \log _{10}$ and $7.22 \pm 1.6 \log _{10}$ of cells $g^{-1}$ in the IVS-1 and IVS-1 + GOS groups, respectively. BB-12 was detected at absolute numbers of $5.83 \pm 0.7 \log _{10}$ and $6.11 \pm 0.7 \log _{10}$ cells $g^{-1}$ in the BB-12 and BB-12+GOS groups, respectively. Comparisons of cell numbers of the probiotic strains among treatments (Fig. 2b) revealed that IVS-1 was detected at significantly higher numbers in fecal samples than BB-12 in both probiotic-only treatments $(P=$ $0.0056)$, and when GOS was added $(P=0.0127)$. Although GOS led to a modest increase in cell numbers of both strains when compared with the probiotic-only groups (Fig. 2b), this increase did not reach statistical significance $(P=0.6682$ and $P=0.3034$, respectively).

\section{Impact of treatments on total number of bifidobacteria in fecal samples}

Genus-specific qPCR was used to determine total fecal bifidobacteria in all six groups (Fig. 3a). There was no significant difference in the numbers of Bifidobacterium in the baseline samples between groups. Among the probiotic treatments, only IVS-1 administration led to an increase in the total number of bifidobacteria $(P=0.0017)$. Significant increases were also detected for all three groups containing GOS when compared to baseline, confirming the bifidogenic effect of GOS that has been observed in previous human studies [40, 41, 45, 78-80].

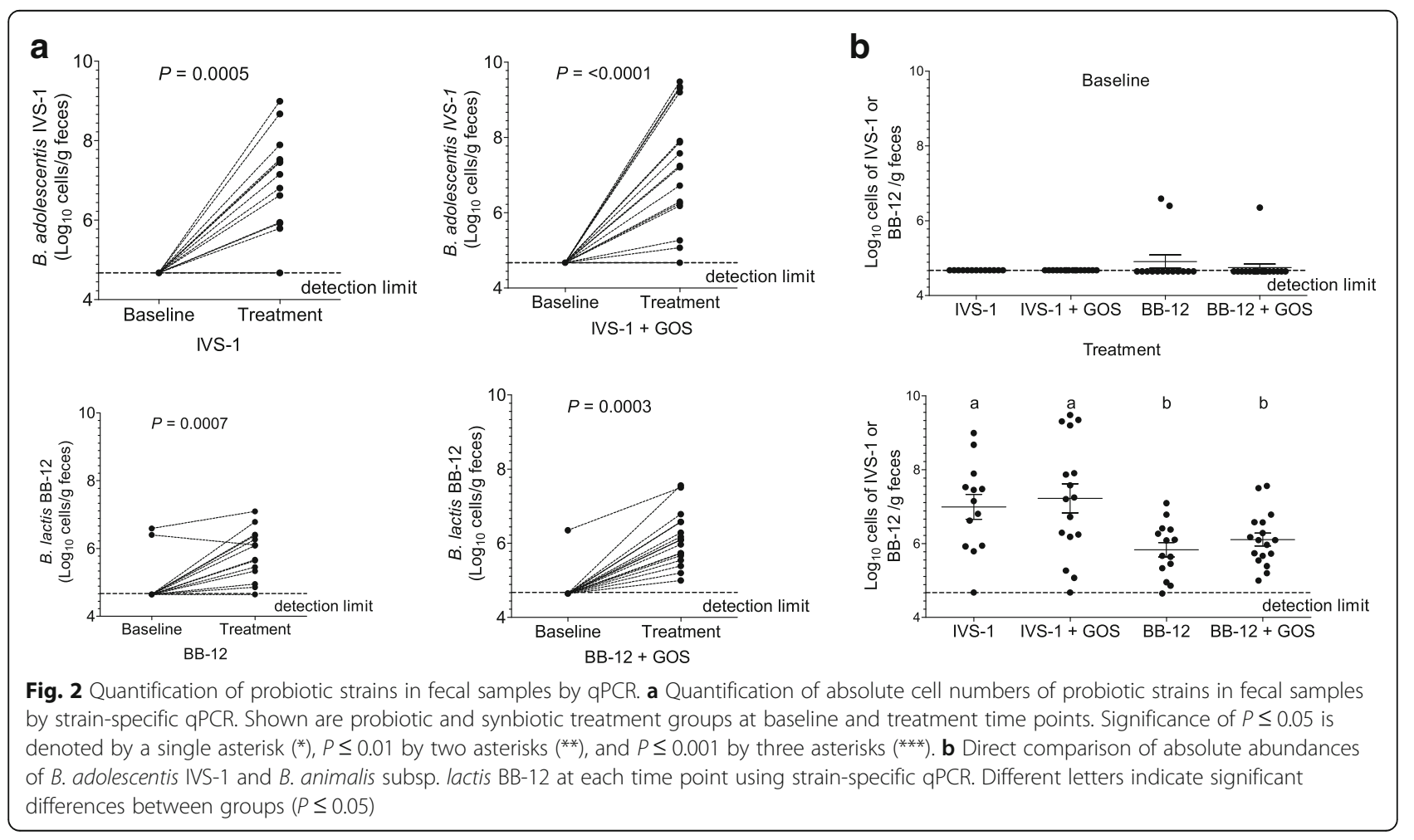




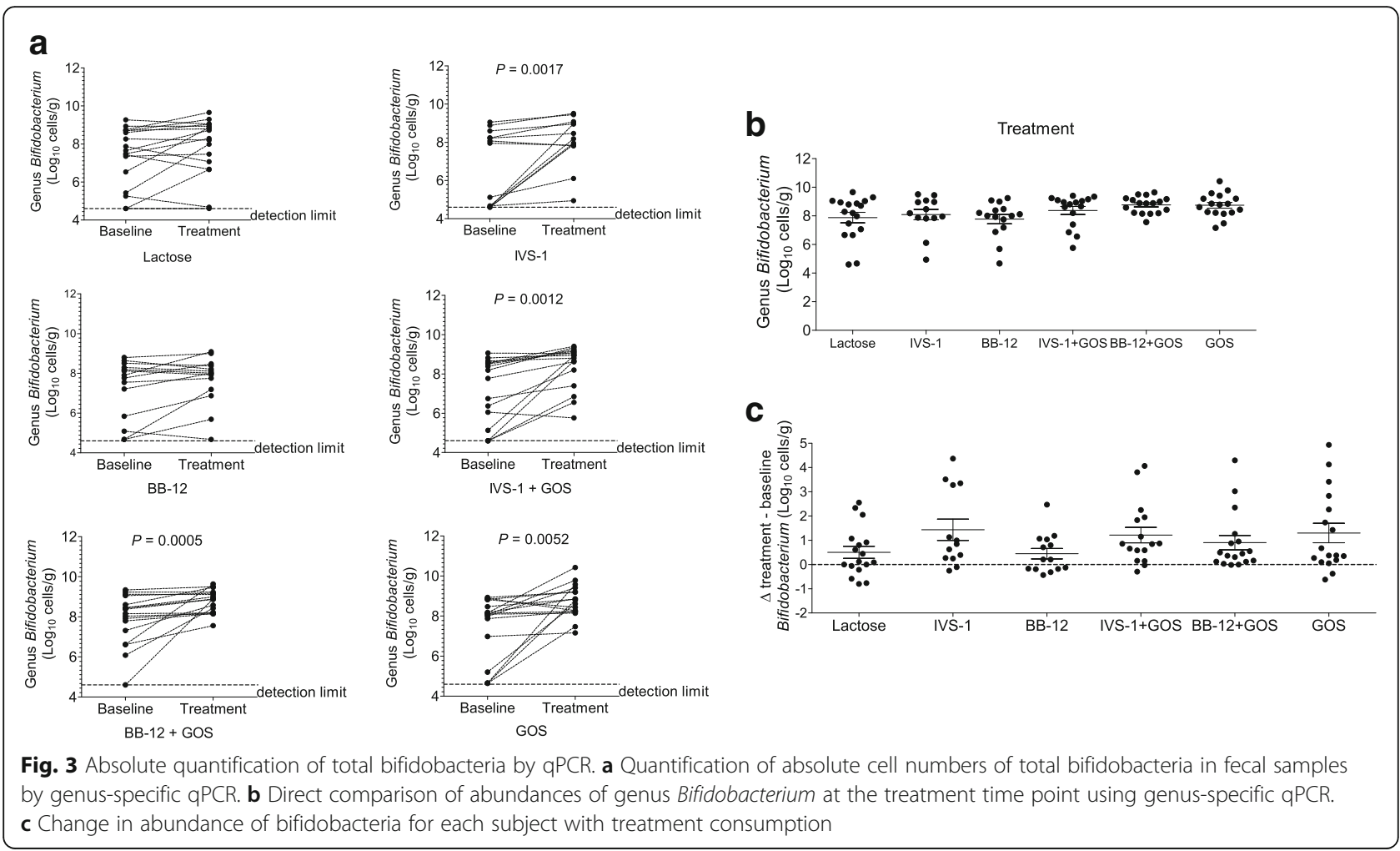

There was no significant difference in the average numbers of Bifidobacterium between treatment groups at the end of the treatment period (Fig. 3b). However, the Bifidobacterium numbers at the baseline varied greatly between individuals within each group, ranging from the detection limit ( $\log _{10} 4.7$ cells $\mathrm{g}^{-1}$ feces) to a maximum of 10.4 $\log _{10}$ cells $\mathrm{g}^{-1}$ feces. In addition, a large variation in the increase in total bifidobacteria was detected among participants (Fig. 3a), suggesting an individualized response to the treatments in all six groups. Therefore, the absolute changes in Bifidobacterium cell numbers for individual subjects were calculated and these shifts compared among the six groups (Fig. 3c). Although there were no significant differences $(P=0.2071)$, the highest increases in Bifidobacterium numbers were found in the IVS-1 group with $1.43 \pm 1.6$ $\log _{10}$, followed by the GOS group with $1.30 \pm 1.7 \log _{10}$. Interestingly, the lactose group also showed a modest increase in bifidobacteria $\left(0.50 \pm 1.0 \log _{10}\right)$.

\section{Characterization of the fecal microbiota by $16 \mathrm{~S}$ rRNA gene sequencing}

Illumina sequencing of $16 \mathrm{~S}$ rRNA gene amplicons was used to determine the effect of the treatments on the overall bacterial community in fecal samples. This analysis revealed that the treatments did not exert a community-wide effect on the resident gut microbial community: Both alpha- and beta-diversity were compared between time points within each group, and across all six groups for both time points, but no differences were detected for any of the comparisons $(P>0.05$, data not shown).

Illumina sequencing, however, did show that the relative abundances of specific taxa were affected by treatments. In particular, the phylum, Actinobacteria, were significantly higher in subjects treated with IVS-1 $(P=0.0072$, $Q=0.0316)$, IVS-1 + GOS $(P=0.0015, Q=0.0176), \mathrm{BB}-12$ $+\operatorname{GOS}(P=0.0279, Q=0.0516)$, GOS $(P=0.0468, Q=$ $0.0722)$, and lactose $(P=0.0526, Q=0.0794)$ (Table 1). At the genus level, the relative abundance of Bifidobacterium was increased in groups IVS-1 $(P=0.0138, Q=0.0420)$, IVS-1 + GOS $(P=0.0039, Q=0.0253), \mathrm{BB}-12+\mathrm{GOS}(P=$ 0.0140, $Q=0.0420)$, and $\operatorname{GOS}(P=0.0686, Q=0.0991)$.

The most significant and consistent shifts were detected among the operational taxonomic units (OTUs) that represented the probiotic strains. The OTU with an identical sequence to $B$. adolescentis IVS-1 (OTU_1) was significantly enriched in groups IVS-1 $(P=0.0010, Q=$ $0.0176)$ and IVS-1 + GOS $(P=0.0176, Q=0.0476)$, indicating that this OTU was primarily enriched through the administration of the probiotic (although non-significant increases were also detected for the GOS and lactose groups). Mann-Whitney test between groups IVS-1 and IVS-1 + GOS showed that IVS-1 + GOS had a significantly higher relative abundance of OTU_1 than IVS-1 $(P=0.0146)$, suggesting a functional synergism between 
Table 1 Proportions of bacterial taxa (with $>0.1 \%$ in at least one of the treatments) significantly influenced by dietary treatments within treatment groups (FDR-adjusted $P$ value $<0.1$ ). Significant values are set in italics

\begin{tabular}{|c|c|c|c|c|c|}
\hline \multicolumn{6}{|l|}{ Treatment } \\
\hline & Taxonomic group & Baseline & Treatment & $P$ value & $\begin{array}{l}\text { Q value } \\
\text { (FDR adjusted) }\end{array}$ \\
\hline \multirow[t]{8}{*}{ IVS-1 } & Phylum & & & & \\
\hline & Actinobacteria & $9.106 \pm 5.25$ & $15.422 \pm 5.98$ & 0.0072 & 0.0316 \\
\hline & Genus & & & & \\
\hline & Bifidobacterium & $7.605 \pm 7.41$ & $14.565 \pm 6.92$ & 0.0138 & 0.0420 \\
\hline & OTUs $^{\mathrm{a}}$ & & & & \\
\hline & OTU_1 (B. adolescentis) & $1.199 \pm 4.24$ & $3.403 \pm 5.08^{*}$ & 0.0010 & 0.0176 \\
\hline & OTU_167 (B. animalis subsp. lactis) & $0.001 \pm 0.00$ & $0.001 \pm 0.00$ & 1.0000 & 1.0000 \\
\hline & OTU_7 (B. adolescentis or ruminantium) & $0.817 \pm 2.24$ & $1.916 \pm 4.33$ & 0.0291 & 0.0516 \\
\hline \multirow[t]{7}{*}{$\mathrm{Bb} 12$} & Phylum & & & & \\
\hline & Actinobacteria & $10.660 \pm 5.17$ & $11.886 \pm 7.68$ & 0.9051 & 0.9540 \\
\hline & Genus & & & & \\
\hline & Bifidobacterium & $8.796 \pm 5.70$ & $9.450 \pm 8.39$ & 0.8782 & 0.9514 \\
\hline & OTUs $^{\mathrm{a}}$ & & & & \\
\hline & OTU_1 (B. adolescentis) & $1.237 \pm 2.46$ & $1.160 \pm 1.62$ & 0.7787 & 0.8814 \\
\hline & OTU_167 (B. animalis subsp. lactis) & $0.034 \pm 0.04$ & $0.055 \pm 0.05$ & 0.0073 & 0.0316 \\
\hline \multirow[t]{11}{*}{ IVS-1 GOS } & Phylum & & & & \\
\hline & Actinobacteria & $8.129 \pm 4.79$ & $15.780 \pm 7.11$ & 0.0015 & 0.0176 \\
\hline & Genus & & & & \\
\hline & Bifidobacterium & $5.959 \pm 5.88$ & $14.650 \pm 7.44$ & 0.0039 & 0.0253 \\
\hline & Anearotrunctus & $0.048 \pm 0.09$ & $0.010 \pm 0.01$ & 0.0236 & 0.0511 \\
\hline & Roseburia & $3.379 \pm 1.64$ & $1.825 \pm 1.15$ & 0.0018 & 0.0176 \\
\hline & OTUs $^{\mathrm{a}}$ & & & & \\
\hline & OTU_1 (B. adolescentis) & $2.476 \pm 4.49$ & $7.344 \pm 7.00^{*}$ & 0.0176 & 0.0476 \\
\hline & OTU_167 (B. animalis subsp. lactis) & $0.001 \pm 0.00$ & $0.003 \pm 0.01$ & 1.0000 & 1.0000 \\
\hline & OTU_102 (Lachnospiracea incertae sedis) & $0.352 \pm 0.49$ & $0.070 \pm 0.16$ & 0.0100 & 0.0390 \\
\hline & OTU_152 (Alistipes) & $0.176 \pm 0.36$ & $0.013 \pm 0.05$ & 0.0463 & 0.0722 \\
\hline \multirow[t]{9}{*}{$\mathrm{Bb} 12 \mathrm{GOS}$} & Phylum & & & & \\
\hline & Actinobacteria & $11.665 \pm 5.90$ & $17.034 \pm 6.51$ & 0.0279 & 0.0516 \\
\hline & Genus & & & & \\
\hline & Bifidobacterium & $8.862 \pm 6.15$ & $15.86 \pm 7.97$ & 0.0140 & 0.0420 \\
\hline & Anaerovorax & $0.295 \pm 0.09$ & $0.18 \pm 0.09$ & 0.0283 & 0.0516 \\
\hline & OTUs $s^{a}$ & & & & \\
\hline & OTU_1 (B. adolescentis) & $3.138 \pm 4.53$ & $6.797 \pm 9.61$ & 0.0812 & 0.1131 \\
\hline & OTU_167 (B. animalis subsp. lactis) & $0.003 \pm 0.001$ & $0.112 \pm 0.18$ & 0.0006 & 0.0176 \\
\hline & OTU_156 (Clostridium XI) & $0.151 \pm 0.20$ & $0.071 \pm 0.21$ & 0.0345 & 0.0585 \\
\hline \multirow[t]{5}{*}{ GOS } & Phylum & & & & \\
\hline & Actinobacteria & $13.923 \pm 8.98$ & $18.067 \pm 9.78$ & 0.0468 & 0.0722 \\
\hline & Genus & & & & \\
\hline & Bifidobacterium & $11.329 \pm 10.48$ & $17.358 \pm 11.41$ & 0.0686 & 0.0991 \\
\hline & Bacteroides & $5.794 \pm 4.43$ & $2.854 \pm 4.11$ & 0.0121 & 0.0420 \\
\hline
\end{tabular}


Table 1 Proportions of bacterial taxa (with $>0.1 \%$ in at least one of the treatments) significantly influenced by dietary treatments within treatment groups (FDR-adjusted $P$ value $<0.1$ ). Significant values are set in italics (Continued)

\begin{tabular}{|c|c|c|c|c|c|}
\hline \multicolumn{6}{|c|}{ Treatment } \\
\hline & Taxonomic group & Baseline & Treatment & $P$ value & $\begin{array}{l}\text { Q value } \\
\text { (FDR adjusted) }\end{array}$ \\
\hline & \multicolumn{5}{|l|}{ OTUs $^{a}$} \\
\hline & OTU_1 (B. adolescentis) & $3.793 \pm 7.47$ & $6.645 \pm 8.83$ & 0.1221 & 0.1587 \\
\hline & OTU_167 (B. animalis subsp. lactis) & $0.000 \pm 0.00$ & $0.203 \pm 0.85$ & 0.4504 & 0.5489 \\
\hline \multirow[t]{10}{*}{ Lactose } & \multicolumn{5}{|l|}{ Phylum } \\
\hline & Actinobacteria & $10.471 \pm 6.75$ & $14.502 \pm 6.89$ & 0.0529 & 0.0794 \\
\hline & \multicolumn{5}{|l|}{ Genus } \\
\hline & Bifidobacterium & $7.895 \pm 8.10$ & $12.008 \pm 8.02$ & 0.0917 & 0.1233 \\
\hline & \multicolumn{5}{|l|}{ OTUs $s^{a}$} \\
\hline & OTU_1 (B. adolescentis) & $1.241 \pm 1.93$ & $2.557 \pm 4.11$ & 0.6562 & 0.7755 \\
\hline & OTU_167 (B. animalis subsp. lactis) & $0.000 \pm 0.00$ & $0.037 \pm 0.14$ & 0.7910 & 0.8814 \\
\hline & OTU_315 (Coprobacillus) & $0.100 \pm 0.09$ & $0.062 \pm 0.06$ & 0.0023 & 0.0179 \\
\hline & OTU_43 (Ruminococcus2) & $0.562 \pm 0.75$ & $0.272 \pm 0.63$ & 0.0051 & 0.0284 \\
\hline & OTU_180 (Bacteroides) & $0.041 \pm 0.17$ & $0.127 \pm 0.40$ & 0.0183 & 0.0476 \\
\hline
\end{tabular}

aff the strain could not be assigned to a type strain (<97\% homology), RDP Classifier was used to determine the most likely genus (80\% cutoff) *Significant difference between the two treatment groups for OTU_1

the synbiotic components IVS-1 and GOS. Similar synergism was not detected for OTU_167 representing $B$. animalis subsp. lactis BB-12 in the BB-12 and GOS groups $(P=0.6895)$. However, this OTU was still significantly increased in both groups when compared to baseline. It was also mostly undetectable in groups that did not receive BB-12 (confirming its allochthonous status in the human gut).

Interestingly, the enrichment of OTU_1 with GOS showed associations with other members of the Bifidobacterium population. An analysis of individuals that had no, or only a low increase of OTU_1 (calculated as relative abundance of OTU_1 of total Bifidobacterium rather than of total bacteria) in groups IVS-1 and IVS-1 + GOS at baseline (non-responders), showed that these subjects had one of six other Bifidobacterium OTUs to be dominant at baseline. The six identified OTUs included OTU_7 (B. adolescentis or ruminantium), OTU_10 (B. longum), OTU_2055 (B. pseudocatenulatum), OTU_2111 (Bifidobacterium sp.), OTU_2202 (B. pseudocatenulatum), and OTU_438 (B. pseudocatenulatum). These OTUs are related to OTU_1 (Additional file 2: Figure S1). None of these six OTUs correlated significantly with OTU_1 when analyzed on their own. However, the sum of the six OTUs correlated negatively with the relative abundance of OTU_1 at the end of the treatment period $(P=0.0313, R=-0.4005)$, indicating competition between these taxa and OTU_1 for the substrate GOS.

Few other changes were detected. We tested an effect of the treatments on specific features of the microbiota that have been suggested to be relevant for health. The ratio between Prevotella and Bacteroides, which has been reported to be influenced by dietary treatments and long-term dietary patterns $[81,82]$ and relevant for metabolic health [81, 83], was not different within treatment groups or when groups were compared (data not shown). Additionally, the relative abundance of butyrate producing genera such as Faecalibacterium, Eubacterium, Roseburia, Lachnobacterium, and Ruminococcus was analyzed. IVS-1 + GOS supplementation significantly decreased the relative abundance of Roseburia (Table 1). We also analyzed the shift in relative abundances (treatment value minus baseline value) and saw a significant reduction of Lachnobacterium $(P<0.0001)$ in the GOS group (data not shown).

\section{Impact of treatments on intestinal permeability and endotoxemia}

To determine the effect of the treatments on intestinal permeability, urine sugar excretion after consumption of a sugar cocktail was compared in pre-treatment samples versus samples taken after the interventions, and LPS and LBP levels were determined in serum. At baseline, no significant differences in gastrointestinal permeability existed between the groups $(P>0.4$, data not shown). No differences in percent change in intestinal permeability were detected between the groups after treatment (inter-group comparisons; Additional file 1: Table S3). When differences in absolute permeability were examined before and after treatment by intra-group comparisons, there were 
significant reductions in permeability for the post-aspirin sucralose:lactulose ratio in groups IVS-1 $(P=0.050)$, IVS-1 + GOS $(P=0.022)$, and GOS $(P=0.010)$ (Fig. 4a). Additionally, there was a significant reduction in the post-aspirin excretion of sucralose in groups BB-12 $(P=0.0020)$ and GOS $(P=0.0171)$ (Fig. 4b). Permeability in the absence of an aspirin challenge was not affected (data not shown).

Baseline markers of endotoxemia did not differ between groups $(P>0.05)$. There were no significant effects of any treatment on serum LPS and LBP levels $(P>0.05$, Additional file 1: Table S4). Minimal changes were seen in both anthropometrics and metabolic markers; the only significant difference was seen between a median increase in HDL-cholesterol in the BB-12 + GOS (3.7 [15.9]) group and a decrease in the BB-12 group $(-3.8[13.2])(P=0.032$, Additional file 1 : Table S5).

\section{Discussion}

In this study, we characterized both the ecological impact and physiological effect of two synbiotic combinations, each composed of a Bifidobacterium strain and the prebiotic GOS, with each other and with their probiotic and prebiotic components. Despite the rational for the synbiotic approach, establishing synergism (ecological or functional) has been challenging for several important reasons [62]. First, most probiotic/prebiotic combinations have been designed and formulated without having demonstrated synergism under the competitive conditions of the gastrointestinal tract. Second, rarely has the experimental design of studies included individual probiotic and synbiotic treatments. Finally, most synbiotic studies used techniques that did not allow for a quantification of the probiotic strain. In this study, we compared synbiotic products comprised of either an in vivo selected autochthonous or an allochthonous strain, each paired with GOS and tested by itself, and included a qPCR analysis with strain level resolution to quantify the probiotic strains.

Consistent with ecological theory [53], the autochthonous strain IVS-1 had a clear ecological advantage over the allochthonous strain BB-12 in that it could be established at about tenfold higher cell numbers. In addition, administration of IVS-1 increased cell numbers of total bifidobacteria as well as the proportion of the genus Bifidobacterium and the phylum Actinobacterium. Such enrichments of higher taxonomic groups through the administration of probiotic bifidobacteria has, to our knowledge, not been previously shown and is remarkable given that the human gut contains high level of resident bifidobacteria and Actinobacteria. In contrast, the allochthonous strain BB-12, although detectable at low levels with $16 \mathrm{~S}$ rRNA gene sequencing, did not increase

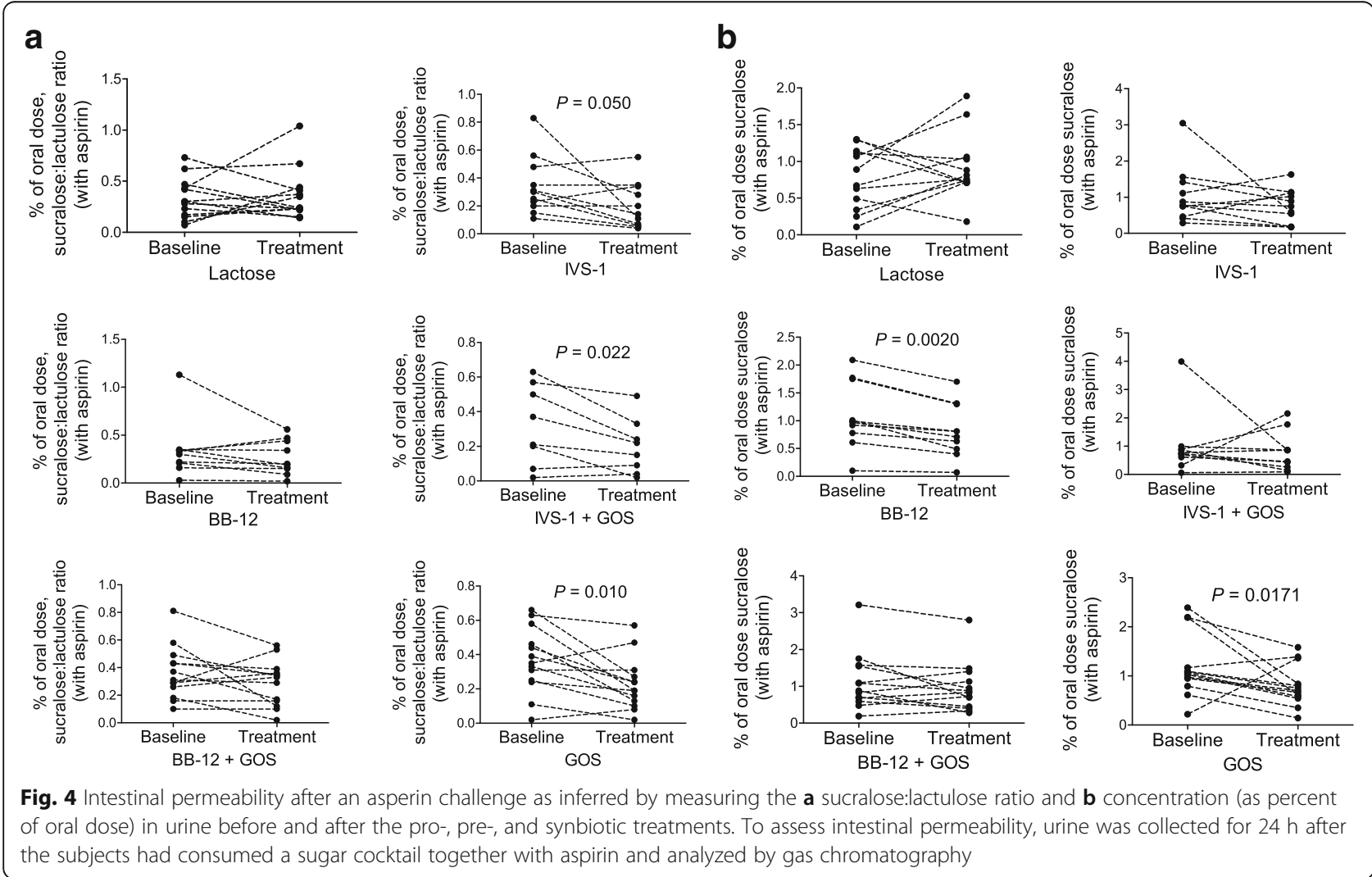


total bifidobacteria. Overall, the findings support the concept that autochthonous members of the microbiota would be substantially more successful when introduced into the human gut $[53,63]$.

The findings on the ability of GOS to support the probiotic strains were more subtle. The OTUs that represented the two probiotic strains (OTU_1 and OTU_167) were higher in the synbiotic groups when compared to the probiotic groups alone, with findings reaching significance for OTU_1 (Table 1). However, cell numbers of both IVS-1 and BB-12 were only marginally and not significantly increased through GOS (Fig. 2). We therefore conclude that although there was a consistent increase in the probiotic strains in the synbiotic groups, inter-individual variability was likely too high to achieve significant findings with the number of subjects in this study. Our findings further suggest that ecological interactions with the resident microbiota but also the specific manner in which the synbiotic preparations were formulated (e.g., all included lactose, and GOS doses were all at $5 \mathrm{~g}$ ) could explain the limited synergism that we observed.

The analysis of the whole gut bacterial community by $16 \mathrm{~S}$ rRNA gene sequencing not only provided insights into the effect of the treatments on overall microbiota composition, but also suggested a possible reason for the limited synbiotic effect that we observed. Overall, the effect of the treatments on gut microbiota composition was remarkably specific, increasing mainly total bifidobacteria and the specific OTUs that represented the probiotic strains, while a limited number of other taxa were decreased (Table 1). In agreement with our previous study [45], the most significant increase induced by GOS was in the genus Bifidobacterium, which occurred in all groups receiving GOS, including the groups that did not receive the probiotic strains. This finding clearly establishes that resident members of the gut microbiota are able to utilize GOS, likely competing with the ingested probiotic strains for the substrate. Accordingly, the OTU representing IVS-1 (OTU_1) correlated negatively with the sum of dominant Bifidobacterium OTUs during treatment with GOS, suggesting competition for the substrate. Given that the gut microbiota is highly specific to an individual, it is likely that resident strains have a higher affinity to GOS compared to the incoming strain even if the latter is autochthonous to humans, as it is still foreign to the microbiota of that specific subject.

Another reason for the limited synergistic effect of GOS might be that the Bifidobacterium strains in the "probiotic only" powders were given as a mixture with lactose, which also served as the placebo in this study. The rational for that choice was that the GOS is synthesized from lactose and contained $22.8 \%$ lactose in the powder that was administered. We further reasoned that lactose would be hydrolyzed and absorbed in lactose-tolerant individuals before it reaches the colon [84]. However, in subjects with insufficient expression of the $L C T$ gene (encoding for lactase) $[85,86]$, lactose can become a "conditional prebiotic" [87] and serve as a colonic substrate for bifidobacteria, which are well equipped to metabolize lactose [88, 89]. While our study subjects self-reported to be lactose tolerant, both the qPCR and amplicon sequencing analyses showed that lactose supplementation did lead to a non-significant and highly personalized increase in the average relative abundance of bifidobacteria. Indeed, seven of the 17 subjects in the lactose group had increases of bifidobacteria of more than $0.50 \log _{10}$ (Fig. 3c). Interestingly, six of these seven "responders" self-identified as African American, who are more likely to carry $L C T$ genes that predispose them to lactose malabsorption $[85,86]$. A mild form of lactose malabsorption was indeed suggested among our subjects by the non-significant increase in passing gas in the lactose group (Additional file 1: Table S5). Therefore, a combination of the "conditional prebiotic" effect of lactose, the ability of the resident microbiota to compete for GOS, and the substantial inter-individual variation might have lowered our ability to detect a more pronounced impact of GOS on establishment of the probiotic strains.

We chose intestinal barrier function as the primary outcome of the human trial because of its importance in the prevention of inflammation not only in metabolic diseases but also in gastrointestinal and systemic inflammatory disorders such as inflammatory bowel disease [90], non-alcoholic steatohepatitis (NASH) [91], and Parkinson disease [92]. In our study, no treatments significantly impacted markers of intestinal permeability in non-aspirin treated subjects. However, when susceptibility to intestinal hyperpermeability was increased through a high-dose of aspirin, permeability was reduced with both probiotic treatments and with GOS intake. While no overall changes in the microbial community were seen, as measured by alpha- and beta-diversity, the enrichment in bifidobacteria common to all of these treatments might be associated with this decrease, and both the autochthonous and the allochthonous probiotic strain showed an effect. As markers of small bowel permeability (5-h urinary lactulose or mannitol; data not shown) were not reduced, the treatment effects appear to be primarily directed to permeability of the colon [75], the site where the majority of bacteria, including bifidobacteria, reside. Overall, our findings are in agreement with observations in rodents that showed that GOS [93], B. adolescentis [32], and B. animalis subsp. lactis CNCM-12494 [94, 95] improved intestinal permeability. In accordance with the findings from the gut microbiota analysis, there was no evidence for synergism in the synbiotic treatments in respect to permeability. Indeed, although permeability (based on sucralose 
excretion) was improved for both the BB-12 and GOS treatments, the BB-12+GOS treatment was not improved. It is possible that the effect was muted when both were present, or that an antagonistic event had occurred, similar to that recently described by Schroeder et al. [96], where mucus permeability was improved by inulin, but not by inulin plus $B$. longum.

Despite the detectable improvement in intestinal permeability, no significant effects on serum endotoxin or metabolic outcomes were seen with any synbiotic, prebiotics, or probiotic treatment. While promising, limited evidence exists to support the use of GOS [97] and bifidobacteria [98] supplementation for serum LPS reduction. In contrast, a synbiotic comprised of Lactobacillus plantarum and FOS was recently shown to significantly reduce sepsis in infants [55]. In other studies, improvements in metabolic markers with GOS intake [41] have been observed, but the impact on metabolic outcomes with bifidobacteria intake is uncertain. Bifidobacteria cell counts were increased through IVS-1 + GOS; however, changes in bifidobacteria are not always associated with improvements in LPS [99] or metabolic markers [100]. It is possible that the treatment duration of 3 weeks was not long enough for metabolic improvements to become evident, or that serum concentrations of these markers were not at high enough concentrations to see a meaningful decrease with treatment. Future clinical trials are warranted, targeting pathologies with an underlying leakiness of the gut, using disease-specific endpoints and longer durations of the treatments. Given that we did not observe synergism between GOS and the probiotic strains, future trials should probably focus on single components, or explore higher doses of GOS in synbiotic combinations.

\section{Conclusion}

This study provided an important test of the synbiotic concept. The findings showed that autochthony of a bacterial strain is more important than the provision of prebiotic substrate at a 5-g dose for the establishment of a probiotic in the human gut. This outcome is likely due to the highly competitive environment that favors autochthonous strains that possess traits that allow colonization while effectively competing for substrates [53]. In addition, although both the probiotic strains and the prebiotic GOS improved barrier function, a combination of the two did not result in apparent synergism. It is unclear how competition for the prebiotic can be avoided, but it is possible that higher doses may be necessary. We selected the daily amount of GOS in this study based on the dose that has previously been shown to induce a bifidogenic effect [39], which was confirmed in this study, and successfully enrich the strain IVS-1 in humans [61]. However, for synbiotic approaches, it might be necessary to give doses of prebiotics that exceed those sufficient for bifidogenic effects to ensure that substrates are available for both the resident microbiota and the incoming microbe. Accordingly, in our previous study in rats, a GOS dose that was approximately 30 -fold higher when adjusted by bodyweight did result in clear synergism and increased competiveness of IVS-1 [61]. Although the synbiotic approach tested in this study did not provide measurable synergism, our findings clearly show that both probiotic strains and the prebiotic improved markers of intestinal permeability. Thus, this report provides a basis for the use of these treatments (or combinations thereof) in pathologies with an underlying leakiness of the gut.

\section{Additional files}

Additional file 1: Table S1. Baseline demographic and metabolic characteristics of study subjects by treatment group. Table S2. Differences in gastrointestinal symptoms by treatment group. Table S3. Percent change in intestinal permeability in subjects by treatment group. Table S4. Differences in markers of endotoxemia by treatment group. Table S5. Percent change in anthropometrics and metabolic markers in subjects by treatment group. (DOCX $49 \mathrm{~kb}$ )

Additional file 2: Figure S1. Phylogenetic analysis of OTU_1 and six closely related Bifidobacterium OTUs that might have competed with OTU_1 for the niche in the GI tract using Maximum Likelihood method. OTU_2281 Lactobacillus animalis was selected as the Outgroup. Numbers indicate branch lengths. (PPTX 41 kb)

\section{Abbreviations}

ALT: Alanine aminotransferase; AST: Aspartate aminotransferase; BMI: Body mass index; CFU: Colony-forming units; Gl: Gastrointestinal;

GOS: Galactooligosaccharide; HDL-C: High-density lipoprotein cholesterol; LAL: Limulus amebocyte lysate; LBP: LPS-binding protein; LDL-C: Low-density lipoprotein cholesterol; LPS: Lipopolysaccharide; OTU: Operational taxonomic unit; TC: Total cholesterol

\section{Acknowledgements}

We thank FrieslandCampina (Amersfoort, the Netherlands) for providing the GOS and Chr. Hansen (Hoersholm, Denmark) for providing Bifidobacterium animalis subsp. lactis BB-12. We also thank Margriet Schoterman (FrieslandCampina) for helpful comments on the manuscript and Inés Martínez for providing technical advice on the bioinformatic analyses.

\section{Funding}

This work was supported by a USDA AFRI Grant \#2012-67017-19344 and by funding from the Nebraska Research Initiative. J.W. acknowledges support through the Campus Alberta Innovation Program.

\section{Availability of data and materials}

The raw 165 rRNA gene sequences have been deposited under the NCBI BioProject ID PRJNA434249, SRA accession: SRP133159. Project information will be accessible (https://www.ncbi.nlm.nih.gov/sra/SRP133159).

\section{Authors' contributions}

JW, RWH, HER, and AK designed the project. HER and KS conducted the human trial and subject recruitment. JAK performed the fecal microbiota analyses and RT-PCR. HER performed the clinical data analyses. JAK and HER correlated the clinical and microbial data. JAK and HER created the figures. JC provided statistical guidance. JW, RWH, HER, and AK supervised and oversaw the research activities. JAK, HER, JW, and RWH wrote the first draft of the manuscript. AK, HER, JW, and RWH provided revisions and thorough editing. All authors helped with the data interpretation and the discussion of the results. All authors read and approved the final manuscript. 


\section{Ethics approval and consent to participate}

This study was approved by the Institutional Review Board at RUMC in February of 2012, and all procedures were conducted according to the principles expressed in the Declaration of Helsinki. This trial was registered at clinicaltrials.gov, identifier NCT02355210 (registered February 4, 2015-retrospectively registered, https://clinicaltrials.gov/ct2/show/ NCT02355210).

\section{Competing interests}

RWH is a member of the Board of Directors of the International Scientific Association for Probiotics and Prebiotics. RWH and JW have received research funding from industry sources involved in the manufacture and marketing of probiotics, prebiotics, and dietary fibers. RWH and JW are co-owners of Synbiotics Solutions, a developer of synbiotic products that involve strain Bifidobacterium adlolescentis IVS-1. The other authors declare that they have no competing interests.

\section{Publisher's Note}

Springer Nature remains neutral with regard to jurisdictional claims in published maps and institutional affiliations.

\begin{abstract}
Author details
'Department of Food Science and Technology, 258 Food Innovation Center Lincoln, University of Nebraska-Lincoln, Lincoln, NE 68588, USA. ${ }^{2}$ Department of Clinical Nutrition, Rush University Medical Center, Chicago, IL, USA. ${ }^{3}$ Division of Digestive Diseases and Nutrition, Department of Internal Medicine, Rush University Medical Center, Chicago, IL, USA. ${ }^{4}$ Department of Biological Sciences, University of Alberta, Edmonton, AB, Canada.

${ }^{5}$ Department of Agricultural, Food, and Nutritional Science, University of Alberta, Edmonton, AB, Canada. ${ }^{6} 4-126 \mathrm{~A}$ Li Ka Shing Centre for Health Research Innovation and 7-142 Katz Group Center, Edmonton, AB T6G 2E1, Canada.
\end{abstract}

\section{Received: 21 February 2018 Accepted: 6 June 2018}

Published online: 28 June 2018

\section{References}

1. Joyce SA, Gahan CGM. The gut microbiota and the metabolic health of the host. Curr Opin Gastroenterol. 2014;30:120-7.

2. Clemente JC, Ursell LK, Parfrey LW, Knight R. The impact of the gut microbiota on human health: an integrative view. Cell. 2012;148:1258-70.

3. Flint HJ, Scott KP, Louis $P$, Duncan SH. The role of the gut microbiota in nutrition and health. Nat Rev Gastroenterol Hepatol. 2012;9:577-89.

4. Marchesi JR, Adams DH, Fava F, Hermes GDA, Hirschfield GM, et al. The gut microbiota and host health: a new clinical frontier. Gut. 2015;65:330-9.

5. Sommer F, Bäckhed F. The gut microbiota - masters of host development and physiology. Nat Rev Microbiol. 2013;11:227-38.

6. Cani PD, Everard A. Talking microbes: when gut bacteria interact with diet and host organs. Mol Nutr Food Res. 2016;60:58-66.

7. Tran CD, Grice DM, Wade B, Kerr CA, Bauer DC, et al. Gut permeability, its interaction with gut microflora and effects on metabolic health are mediated by the lymphatics system, liver and bile acid. Future Microbiol. 2015;10:1339-53.

8. Farhadi A, Banan A, Fields J, Keshavarzian A. Intestinal barrier: an interface between health and disease. J Gastroenterol Hepatol. 2003;18:479-97.

9. Le Chatelier E, Nielsen T, Qin J, Prifti E, Hildebrand F, et al. Richness of human gut microbiome correlates with metabolic markers. Nature. 2013;500:541-6.

10. Esser N, Legrand-Poels S, Piette J, Scheen AJ, Paquot N. Inflammation as a link between obesity, metabolic syndrome and type 2 diabetes. Diabetes Res Clin Pract. 2014;105:141-50.

11. Zeyda M, Stulnig TM. Obesity, inflammation, and insulin resistance-a mini-review. Gerontology. 2009;55:379-86.

12. Cani PD, Amar J, Iglesias MA, Poggi M, Knauf C, et al. Metabolic endotoxemia initiates obesity and insulin resistance. Diabetes. 2007;56:1761-72.

13. Neves AL, Coelho J, Couto L, Leite-Moreira A, Roncon-Albuquerque R. Metabolic endotoxemia: a molecular link between obesity and cardiovascular risk. J Mol Endocrinol. 2013;51:R51-64.

14. Cani PD. Interactions between gut microbes and host cells control gut barrier and metabolism. Int J Obes Suppl. 2016;6:S28-31.
15. Cani PD, Bibiloni R, Knauf C, Waget A, Neyrinck AM, et al. Changes in gut microbiota control metabolic diet-induced inflammation in high-fat dietinduced obesity and diabetes in mice. Diabetes. 2008;57:1470-81.

16. Cani PD, Osto M, Geurts L, Everard A. Involvement of gut microbiota in the development of low-grade inflammation and type 2 diabetes associated with obesity. Gut Microbes. 2012;3:279-88.

17. Akbari P, Braber S, Alizadeh A, Verheijden K, Schoterman M, et al. Galactooligosaccharides protect the intestinal barrier by maintaining the tight junction network and modulating the inflammatory responses after a challenge with the mycotoxin deoxynivalenol in human Caco-2 cell monolayers and B6C3F1 mice. J Nutr. 2015;145:1604-13.

18. Akbari P, Fink-Gremmels J, Willems RHAM, Difilippo E, Schols HA, et al. Characterizing microbiota-independent effects of oligosaccharides on intestinal epithelial cells: insight into the role of structure and size: structure-activity relationships of non-digestible oligosaccharides. Eur J Nutr. 2017:56:1919-30.

19. Alizadeh A, Akbari P, Difilippo E, Schols HA, Ulfman LH, et al. The piglet as a model for studying dietary components in infant diets: effects of galactooligosaccharides on intestinal functions. Br J Nutr. 2015;115:605-18.

20. Madsen K, Cornish A, Soper P, McKaigney C, Jijon H, et al. Probiotic bacteria enhance murine and human intestinal epithelial barrier function. Gastroenterology. 2001;121:580-91.

21. Hsieh C-Y, Osaka T, Moriyama E, Date Y, Kikuchi J, et al. Strengthening of the intestinal epithelial tight junction by Bifidobacterium bifidum. Physiol Rep. 2015;3:e12327.

22. Guo S, Gillingham T, Guo Y, Meng D, Zhu W, Walker WA, et al. Secretions of Bifidobacterium infantis and Lactobacillus acidophilus protect intestinal epithelial barrier function. J Pediatr Gastroenterol Nutr. 2017:64:404-12.

23. Anderson RC, Cookson AL, McNabb WC, Kelly WJ, Roy N. Lactobacillus plantarum DSM 2648 is a potential probiotic that enhances intestinal barrier function. FEMS Microbiol Lett. 2010;309:184-92.

24. Mujagic Z, De Vos P, Boekschoten MV, Govers C, Pieters HJH, De Wit NJ, et al. The effects of Lactobacillus plantarum on small intestinal barrier function and mucosal gene transcription; a randomized double-blind placebo controlled trial. Sci Rep. 2017:4:40128.

25. Ren C, Dokter-Fokkens J, Figueroa Lozano S, Zhang Q, Haan BJ, et al. Lactic acid bacteria may impact intestinal barrier function by modulating goblet cells. Mol Nutr Food Res. 2018:62:1700572.

26. Cani PD, Neyrinck AM, Fava F, Knauf C, Burcelin RG, et al. Selective increases of bifidobacteria in gut microflora improve high-fat-diet-induced diabetes in mice through a mechanism associated with endotoxaemia. Diabetologia. 2007:50:2374-83.

27. Cani PD, Delzenne NM. Interplay between obesity and associated metabolic disorders: new insights into the gut microbiota. Curr Opin Pharmacol. 2009; 9:737-43.

28. Mills DA. Probiotic nomenclature matters redux: confusion on Bifidobacterium longum subsp. infantis taxonomy persists. Curr Med Res Opin. 2017;33:2097.

29. Ewaschuk JB, Diaz H, Meddings L, Diederichs B, Dmytrash A, et al. Secreted bioactive factors from Bifidobacterium infantis enhance epithelial cell barrier function. Am J Physiol Gastrointest Liver Physiol. 2008;295:G1025-34.

30. Bergmann KR, Liu SXL, Tian R, Kushnir A, Turner JR, et al. Bifidobacteria stabilize claudins at tight junctions and prevent intestinal barrier dysfunction in mouse necrotizing enterocolitis. Am J Pathol. 2013;182:1596-606.

31. Griffiths EA, Duffy LC, Schanbacher FL, Qiao H, Dryja D, et al. In vivo effects of bifidobacteria and lactoferrin on gut endotoxin concentration and mucosal immunity in balb/c mice. Dig Dis Sci. 2004;49:579-89.

32. Wang Z, Xiao G, Yao Y, Guo S, Lu K, et al. The role of bifidobacteria in gut barrier function after thermal injury in rats. J Trauma-Injury Infect Crit Care. 2006;61:650-7.

33. Chen J, Wang RR-L, Li X-F, Wang RR-L. Bifidobacterium adolescentis supplementation ameliorates visceral fat accumulation and insulin sensitivity in an experimental model of the metabolic syndrome. Br J Nutr. 2012;107:1429-34.

34. Reichold A, Brenner SA, Spruss A, Förster-Fromme K, Bergheim I, et al. Bifidobacterium adolescentis protects from the development of nonalcoholic steatohepatitis in a mouse model. J Nutr Biochem. 2014:25:118-25.

35. Martínez I, Wallace G, Zhang C, Legge R, Benson AK, et al. Diet-induced metabolic improvements in a hamster model of hypercholesterolemia are strongly linked to alterations of the gut microbiota. Appl Environ Microbiol. 2009;75:4175-84.

36. Fanaro S, Marten B, Bagna R, Vigi V, Fabris C, et al. Galacto-oligosaccharides are bifidogenic and safe at weaning: a double-blind randomized multicenter study. J Pediatr Gastroenterol Nutr. 2009;48:82-8. 
37. Sierra C, Bernal M-J, Blasco J, Martinez R, Dalmau J, et al. Prebiotic effect during the first year of life in healthy infants fed formula containing GOS as the only prebiotic: a multicentre, randomised, double-blind and placebocontrolled trial. Eur J Nutr. 2015:54:89-99.

38. Haarman M, Knol J. Quantitative real-time PCR analysis of fecal Lactobacillus species in infants receiving a prebiotic infant formula. Appl Environ Microbiol. 2006;72:2359-65.

39. Davis LMG, Martínez I, Walter J, Hutkins R. A dose dependent impact of prebiotic galactooligosaccharides on the intestinal microbiota of healthy adults. Int J Food Microbiol. 2010;144:285-92.

40. Walton GE, van den Heuvel EGHM, Kosters MHW, Rastall RA, Tuohy KM, et al. A randomised crossover study investigating the effects of galactooligosaccharides on the faecal microbiota in men and women over 50 years of age. Br J Nutr. 2012;107:1466-75.

41. Vulevic J, Juric A, Tzortzis G, Gibson GR. A mixture of trans-galactooligosaccharides reduces markers of metabolic syndrome and modulates the fecal microbiota and immune function of overweight adults. J Nutr. 2013:324-31.

42. Bouhnik $Y$, Raskine L, Simoneau G, Paineau D, Bornet F. The capacity of short-chain fructo-oligosaccharides to stimulate faecal bifidobacteria: a dose-response relationship study in healthy humans. Nutr J. 2006;5:8.

43. Ramirez-Farias C, Slezak K, Fuller Z, Duncan A, Holtrop G, et al. Effect of inulin on the human gut microbiota: stimulation of Bifidobacterium adolescentis and Faecalibacterium prausnitzii. Br J Nutr. 2008;101:541-50.

44. Clarke ST, Brooks SP, Inglis GD, Yanke L, Green J, Petronella N., Ramdath DD, Bercik P, Green-Johnson JM, Kalmokoff M. Impact of $\beta 2-1$ fructan on faecal community change: results from a placebo-controlled, randomised, doubleblinded, cross-over study in healthy adults. Br J Nutr. 2017;118:441-53.

45. Davis L, Martínez I, Walter J, Goin C, Hutkins RW. Barcoded pyrosequencing reveals that consumption of galactooligosaccharides results in a highly specific bifidogenic response in humans. PLoS One. 2011;6:e25200.

46. Martínez I, Kim J, Duffy PR, Schlegel VL, Walter J. Resistant starches types 2 and 4 have differential effects on the composition of the fecal microbiota in human subjects. PLoS One. 2010;5:e15046.

47. Ringel-Kulka T, Cheng J, Ringel Y, Salojärvi J, Carroll I, et al. Intestina microbiota in healthy U.S. young children and adults - a high throughput microarray analysis. PLoS One. 2013;8:e64315.

48. Agans R, Rigsbee L, Kenche H, Michail S, Khamis HJ, et al. Distal gut microbiota of adolescent children is different from that of adults. FEMS Microbiol Ecol. 2011;77:404-12.

49. Salonen A, Lahti L, Salojärvi J, Holtrop G, Korpela K, et al. Impact of diet and individual variation on intestinal microbiota composition and fermentation products in obese men. ISME J. 2014;8:2218-30.

50. Venkataraman A, Sieber JR, Schmidt AW, Waldron C, Theis KR, et al. Variable responses of human microbiomes to dietary supplementation with resistant starch. Microbiome. 2016;4:33.

51. Kolida S, Gibson GR. Synbiotics in health and disease. Annu Rev Food Sci Technol. 2011:2:373-93.

52. Mallon CA, Van Elsas JD, Salles JF. Microbial invasions: the process, patterns, and mechanisms. Trends Microbiol. 2015;23:719-29.

53. Walter J, Maldonado-Gómez MX, Martínez I. To engraft or not to engraft: an ecological framework for gut microbiome modulation with live microbes. Curr Opin Biotechnol. 2017;49:129-39.

54. Freitas MB, Moreira EAM, de Lima Oliveira D, Tomio C, Rosa JS, Moreno YMF, et al. Effect of synbiotic supplementation in children and adolescents with cystic fibrosis: a randomized controlled clinical trial. Eur J Clin Nutr.2018;72:736-43.

55. Panigrahi P, Parida S, Nanda NC, Satpathy R, Pradhan L, et al. A randomized synbiotic trial to prevent sepsis among infants in rural India. Nature. Epub ahead of print 2017. https://doi.org/10.1038/nature23480.

56. Rossi M, Johnson DW, Morrison M, Pascoe EM, Coombes JS, et al. Synbiotics easing renal failure by improving gut microbiology (SYNERGY): a randomized trial. Clin J Am Soc Nephrol. 2016;11:223-31.

57. Ogawa T, Asai Y, Yasuda K, Sakamoto H. Oral immunoadjuvant activity of a new synbiotic Lactobacillus casei subsp casei in conjunction with dextran in BALB/c mice. Nutr Res. 2005;25:295-304.

58. Tanaka R, Takayama H, Morotomi M, Kuroshima T, Ueyama S, et al. Effects of administration of TOS and Bifidobacterium breve 4006 on the human fecal flora. Bifidobact Microflora. 1983;2:17-24.

59. Pietro FA, Luceri C, Dolara P, Giannini A, Biggeri A, et al. Antitumorigenic activity of the prebiotic inulin enriched with oligofructose in combination with the probiotics Lactobacillus rhamnosus and Bifidobacterium lactis on azoxymethaneinduced colon carcinogenesis in rats. Carcinogenesis. 2002;23:1953-60.
60. Wang X, Brown IL, Evans AJ, Conway PL. The protective effects of high amylose maize (amylomaize) starch granules on the survival of Bifidobacterium spp. in the mouse intestinal tract. J Appl Microbiol. 1999;87:631-9.

61. Krumbeck JA, Maldonado-Gomez MX, Martínez I, Frese SA, Burkey TE, et al. In vivo selection to identify bacterial strains with enhanced ecological performance in synbiotic applications. Appl Environ Microbiol. 2015;81: 2455-65.

62. Krumbeck JA, Walter J, Hutkins RW. Synbiotics for improved human health: recent developments, challenges, and opportunities. Annu Rev Food Sci Technol. 2018;9:451-79.

63. Maldonado-Gómez MX, Martínez I, Bottacini F, O'Callaghan A, Ventura M, et al. Stable engraftment of Bifidobacterium longum AH1206 in the human gastrointestinal tract depends on individualized features of the resident microbiome. Cell Host Microbe. 2016;20:515-26.

64. Bindels LB, Munoz RRS, Gomes-Neto JC, Mutemberezi V, Martínez I, Salazar $\mathrm{N}$, et al. Resistant starch can improve insulin sensitivity independently of the gut microbiota. Microbiome. 2017;5:12.

65. Wu RY, Määttänen P, Napper S, Scruten E, Li B, et al. Non-digestible oligosaccharides directly regulate host kinome to modulate host inflammatory responses without alterations in the gut microbiota. Microbiome. 2017:5:135

66. Fransen F, Sahasrabudhe NM, Elderman M, Bosveld M, El Aidy S, Hugenholtz $F$, et al. $\beta 2 \rightarrow 1$-fructans modulate the immune system in vivo in a microbiota-dependent and-independent fashion. Front Immunol. 2017;8:154.

67. Pokusaeva K, Fitzgerald GF, Sinderen D. Carbohydrate metabolism in Bifidobacteria. Genes Nutr. 2011;6:285.

68. Vernazza CL, Gibson GR, Rastall R. Carbohydrate preference, acid tolerance and bile tolerance in five strains of Bifidobacterium. J Appl Microbiol. 2006; 100:846-53.

69. Thongaram T, Hoeflinger JL, Chow J, Miller MJ. Prebiotic galactooligosaccharide metabolism by probiotic lactobacilli and bifidobacteria. J Agric Food Chem. 2017:65:4184-92

70. Alander M, Mättö J, Kneifel W, Johansson M, Koegle B, et al. Effect of galacto-oligosaccharide supplementation on human faecal microflora and on survival and persistence of Bifidobacterium lactis $\mathrm{Bb}-12$ in the gastrointestinal tract. Int Dair. 2001;11:817-25.

71. Malinen E, Mättö J, Salmitie M, Alander M, Saarela M, et al. PCR-ELISA II: analysis of Bifidobacterium populations in human faecal samples from a consumption trial with Bifidobacterium lactis $\mathrm{Bb}-12$ and a galactooligosaccharide preparation. Syst Appl Microbiol. 2002;25:249-58.

72. Satokari RM, Vaughan EE, Akkermans AD, Saarela M, de Vos WM. Polymerase chain reaction and denaturing gradient gel electrophoresis monitoring of fecal Bifidobacterium populations in a prebiotic and probiotic feeding trial. Syst Appl Microbiol. 2001;231:227-31.

73. Weiss S, Xu ZZ, Peddada S, Amir A, Bittinger K, et al. Normalization and microbial differential abundance strategies depend upon data characteristics. Microbiome. 2017;5:1-18

74. Taipale $T$, Pienihäkkinen $\mathrm{K}$, Isolauri $\mathrm{E}$, Larsen $\mathrm{C}$, Brockmann $\mathrm{E}$, et al. Bifidobacterium animalis subsp. lactis BB-12 in reducing the risk of infections in infancy. Br J Nutr. 2010;105:409-16.

75. Shaikh M, Rajan K, Forsyth CB, Voigt RM, Keshavarzian A. Simultaneous gaschromatographic urinary measurement of sugar probes to assess intestinal permeability: use of time course analysis to optimize its use to assess regional gut permeability. Clin Chim Acta. 2015;10:24-32.

76. Farhadi A, Gundlapalli S, Shaikh M, Frantzides C, Harrell L, et al. Susceptibility to gut leakiness: a possible mechanism for endotoxaemia in non-alcoholic steatohepatitis. Liver Int. 2008;28:1026-33.

77. Hilsden R, Meddings J, Sutherland L. Intestinal permeability changes in response to acetylsalicylic acid in relatives of patients with Crohn's disease. Gastroenterology. 1996;110:1395-403.

78. Ben X-M, Li J, Feng Z-T, Shi S-Y, Lu Y-D, et al. Low level of galactooligosaccharide in infant formula stimulates growth of intestinal bifidobacteria and lactobacilli. World J Gastroenterol. 2008;14:6564-8.

79. Vulevic J, Drakoularakou A, Yaqoob P, Tzortzis G, Gibson GR. Modulation of the fecal microflora profile and immune function by a novel transgalactooligosaccharide mixture (B-GOS) in healthy elderly volunteers. Am J Clin Nutr. 2008:88:1438-46.

80. Chandel D, Perez-Munoz M, Yu F, Boissy R, Satpathy R, et al. Changes in the gut microbiota after early administration of oral synbiotics to young infants in India. J Pediatr Gastroenterol Nutr. 2017;65:218-24. 
81. Kovatcheva-Datchary P, Nilsson A, Akrami R, Lee YS, De Vadder F, et al. Dietary fiber-induced improvement in glucose metabolism is associated with increased abundance of Prevotella. Cell Metab. 2015;22:971-82.

82. Wu GD, Chen J, Hoffmann C, Bittinger $K$, Chen $Y$, et al. Linking long-term dietary patterns with gut microbial enterotypes. Science (80- ). 2011;334:105-9.

83. Hjorth MF, Roager HM, Larsen TM, Poulsen SK, Licht TR, et al. Pretreatment microbial Prevotella-to-Bacteroides ratio, determines body fat loss success during a 6-month randomized controlled diet intervention. Int J Obes. 2018:42:580-3.

84. Angelakis E, Lagier J-C. Samples and techniques highlighting the links between obesity and microbiota Microb Pathog Epub ahead of print 2016. https://doi.org/10.1016/j.micpath.2016.01.024.

85. Blekhman R, Goodrich JK, Huang K, Sun Q, Bukowski R, et al. Host genetic variation impacts microbiome composition across human body sites. Genome Biol. 2015;16:191.

86. Tishkoff SA, Reed FA, Ranciaro A, Voight BF, Courtney C, et al. Convergent adaptation of human lactase persistence in Africa and Europe. Nat Genet. 2007:39:31-40

87. Szilagyi A. Redefining lactose as a conditional prebiotic. Can J Gastroenterol. 2004;18:163-7.

88. Parche S, Jacobs D, Arigoni F, Titgemeyer F, Jankovic I. Lactose-over-glucose preference in Bifidobacterium longum NCC2705: glcP, encoding a glucose transporter, is subject to lactose repression. J Bacteriol. 2006;188:1260-5.

89. Azcarate-Peril MA, Ritter AJ, Savaiano D, Monteagudo-Mera A, Anderson C, Magness ST, Klaenhammer TR. Impact of short-chain galactooligosaccharides on the gut microbiome of lactose-intolerant individuals. PNAS. 2017:114:E367-75.

90. Antoni L, Nuding S, Wehkamp J, Stange EF. Intestinal barrier in inflammatory bowel disease. World J Gastrointerol. 2014;20:1165-79.

91. Scarpellini E, Lupo M, legri C, Gasbarrini A, De Santis A, et al. Intestinal permeability in non-alcoholic fatty liver disease: the gut-liver axis. Rev Recent Clin Trials. 2014;9:141-7.

92. Forsyth C, Shannon K, Kordower J, Voigt R, Shaikh M, et al. Increased intestinal permeability correlates with sigmoid mucosa alpha-synuclein staining and endotoxin exposure markers in early Parkinson's disease. PLoS One. 2011:6:e28032.

93. Zhong Y, Cai D, Cai W, Geng S, Chen L, et al. Protective effect of galactooligosaccharide-supplemented enteral nutrition on intestinal barrier function in rats with severe acute pancreatitis. Clin Nutr. 2009;28:575-80.

94. Martín R, Laval L, Chain F, Miquel S, Natividad J, et al. Bifidobacterium animalis ssp. lactis CNCM-12494 restores gut barrier permeability in chronically low-grade inflamed mice. Front Microbiol. 2016;7:1-12.

95. Agostini S, Goubern M, Tondereau V, Salvador-Cartier C, Bezirard V, et al. A marketed fermented dairy product containing Bifidobacterium lactis CNCM I-2494 suppresses gut hypersensitivity and colonic barrier disruption induced by acute stress in rats. Neurogastroenterol Motil. 2012;24:376-e172.

96. Schroeder B, Birchenough GM, Stahlman M, Arike L, Johansson ME, et al. Bifidobacteria or fiber protects against diet-induced microbiota-mediated colonic mucus deterioration. Cell Host Microbe. 2018;23:27-40.e7.

97. Morel F, Dai Q, Ni J, Thomas D, Parnet P, et al. a-Galacto-oligosaccharides dose-dependently reduce appetite and decrease inflammation in overweight adults. J Nutr. 2015;145:2052-9.

98. Rodes L, Saha S, Tomaro-Duchesneau C, Prakash S. Microencapsulated Bifidobacterium longum subsp. infantis ATCC 15697 favorably modulates gut microbiota and reduces circulating endotoxins in F344 rats. Biomed Res Int. 2014;602832. https://www.hindawi.com/journals/bmri/2014/602832/abs/.

99. Canfora EE, van der Beek, Christina M Hermes GDA, Gijs GH, Jocken JWE, Holst JJ, et al. Supplementation of diet with galacto-oligosaccharides increases bifidobacteria, but not insulin sensitivity, in obese prediabetic individuals. Gastroenterology. 2017;153:87-97.e3.

100. Carvalho-Wells AL, Helmolz K, Nodet C, Molzer C, Leonard C, et al. Determination of the in vivo prebiotic potential of a maize-based whole grain breakfast cereal: a human feeding study. Br J Nutr. 2010;104:1353-6.

\section{Ready to submit your research? Choose BMC and benefit from:}

- fast, convenient online submission

- thorough peer review by experienced researchers in your field

- rapid publication on acceptance

- support for research data, including large and complex data types

- gold Open Access which fosters wider collaboration and increased citations

- maximum visibility for your research: over $100 \mathrm{M}$ website views per year

At BMC, research is always in progress.

Learn more biomedcentral.com/submissions 